\title{
Fourth-Order Compact Difference Schemes for the Riemann-Liouville and Riesz Derivatives
}

\author{
Yuxin Zhang, ${ }^{1}$ Hengfei Ding, ${ }^{1}$ and Jincai Luo ${ }^{2}$ \\ ${ }^{1}$ School of Mathematics and Statistics, Tianshui Normal University, Tianshui 741001, China \\ ${ }^{2}$ Caoba Nine-Year School, Honghe Country, Lixian 742203, China
}

Correspondence should be addressed to Yuxin Zhang; zhangyuxin2006@163.com

Received 4 March 2014; Revised 14 April 2014; Accepted 14 April 2014; Published 30 April 2014

Academic Editor: Robert A. Van Gorder

Copyright (c) 2014 Yuxin Zhang et al. This is an open access article distributed under the Creative Commons Attribution License, which permits unrestricted use, distribution, and reproduction in any medium, provided the original work is properly cited.

We propose two new compact difference schemes for numerical approximation of the Riemann-Liouville and Riesz derivatives, respectively. It is shown that these formulas have fourth-order convergence order by means of the Fourier transform method. Finally, some numerical examples are implemented to testify the efficiency of the numerical schemes and confirm the convergence orders.

\section{Introduction}

Nowadays, fractional derivatives are used to model various different phenomena in science and engineering, such as physics, materials, control theory, biology, and finance [1-3]. Therefore, to obtain highly accurate numerical methods for the fractional derivatives is of great importance, but, due to the nonlocal property of the fractional derivatives, a lot of improvements remain in the present numerical approaches.

As we all know, there are several different ways to define the fractional derivatives, and the most commonly used fractional derivatives are Grünwald-Letnikov derivative, Riemann-Liouville derivative, Riesz derivative, and Caputo derivative. Accordingly, there are a lot of different numerical methods for the above fractional derivatives [4-14]. In this paper, we propose two high-order numerical formulas for the Riemann-Liouville and Riesz derivatives.

The plan of the remainder of this paper is as follows. In Section 2, we introduce the preliminary knowledge for the rest of the paper. Two novel numerical schemes for the Riemann-Liouville and Riesz derivatives are proposed in Section 3. In Section 4, some numerical experiments are carried out to support the theoretical analysis. Finally, the paper concludes with a summary drawn in Section 5.

\section{The Preliminary Knowledge}

In this section, we will introduce the definitions of the Riemann-Liouville and Riesz derivatives and a lemma used throughout the remaining sections of the paper.

Definition 1 (see [15]). The $\alpha$ order left and right RiemannLiouville derivatives of the function $f(x)$ are defined as

$$
\begin{array}{r}
{ }_{\mathrm{RL}} D_{-\infty, x}^{\alpha} f(x)=\frac{1}{\Gamma(n-\alpha)}\left(\frac{d}{d x}\right)^{n} \int_{-\infty}^{x} \frac{f(t)}{(x-t)^{\alpha-n+1}} d t, \\
n-1 \leq \alpha<n \in \mathbb{Z}^{+}, \\
\mathrm{RL}_{x,+\infty} D_{x(x)}^{\alpha}=\frac{1}{\Gamma(n-\alpha)}\left(-\frac{d}{d x}\right)^{n} \int_{x}^{+\infty} \frac{f(t)}{(t-x)^{\alpha-n+1}} d t, \\
n-1 \leq \alpha<n \in \mathbb{Z}^{+} .
\end{array}
$$

Definition 2 (see [15]). The $\alpha$ order Riesz derivative of the function $f(x)$ is defined as

$$
\frac{\partial^{\alpha} f(x)}{\partial|x|^{\alpha}}=-\frac{1}{2 \cos (\pi \alpha / 2)}\left({ }_{\mathrm{RL}} D_{-\infty, x}^{\alpha}+{ }_{\mathrm{RL}} D_{x,+\infty}^{\alpha}\right) f(x) .
$$


Lemma 3 (see [16]). Let $\alpha>0, f \in C_{0}^{\infty}(\mathbb{R})$; the Fourier transform of the left Riemann-Liouville-fractional derivative is

$$
\mathscr{F}\left({ }_{R L} D_{-\infty, x}^{\alpha} f(x), \omega\right)=(i \omega)^{\alpha} \widehat{f}(\omega),
$$

where $\widehat{f}(\omega)$ denotes the Fourier transform of $f(x)$; that is,

$$
\widehat{f}(\omega)=\int_{\mathbb{R}} \exp (-i \omega x) f(x) d x
$$

\section{New Numerical Formulas for the Riemann- Liouville and Riesz Derivatives}

Firstly, we divide the given interval $[a, b]$ into

$$
\Lambda:-\infty \leq a=x_{0}<x_{1}<\cdots<x_{m}=b \leq \infty
$$

and $x_{l}=a+l h$, in which $h=(b-a) / m, l=0,1, \ldots, m$.

In [17], Tuan and Gorenflo introduced the following fractional center difference operator:

$$
{ }_{C} \Delta_{h}^{\alpha} f(x)=\sum_{j=0}^{\infty}(-1)^{j}\left(\begin{array}{c}
\alpha \\
j
\end{array}\right) f\left(x-\left(j-\frac{\alpha}{2}\right) h\right) .
$$

It is proved that it has second-order accuracy for the Riemann-Liouville derivative. Inspired by above fractional center difference operator, now, we give a fourth-order approximation formula for the Riemann-Liouville derivative by the following theorem.

Theorem 4. Let $f(x)$ and the Fourier transform of the ${ }_{R L} D_{-\infty, x}^{\alpha+4} f(x)$ all belong to $L_{1}(\mathbb{R})$. Then, one has

$$
{ }_{R L} D_{-\infty, x}^{\alpha} f(x)=\frac{1}{h^{\alpha}} \frac{C^{\Delta_{h}^{\alpha}} f(x)}{1+(\alpha / 24) \delta_{x}^{2}}+\mathcal{O}\left(h^{4}\right),
$$

uniformly for $x \in \mathbb{R}$, where $\delta_{x}^{2}$ is the second-order central difference operator and defied as $\delta_{x}^{2} f_{j}=f_{j+1}-2 f_{j}+f_{j-1}$.

Proof. Taking the Fourier transform to fractional center difference operator ${ }_{C} \Delta_{h}^{\alpha}$ and getting

$$
\begin{aligned}
& \mathscr{F}\left\{\frac{C^{\Delta_{h}^{\alpha} f(x)}}{h^{\alpha}}, \omega\right\} \\
&= \frac{1}{h^{\alpha}} \sum_{j=0}^{\infty}(-1)^{j}\left(\begin{array}{c}
\alpha \\
j
\end{array}\right) \exp \left(-i \omega\left(j-\frac{\alpha}{2}\right) h\right) \widehat{f}(\omega) \\
&=\frac{1}{h^{\alpha}}\left(\sum_{j=0}^{\infty}(-1)^{j}\left(\begin{array}{c}
\alpha \\
j
\end{array}\right) \exp (-i j \omega h)\right) \exp \left(\frac{1}{2} i \omega \alpha h\right) \hat{f}(\omega) \\
&=(i \omega)^{\alpha} \widehat{f}(\omega)\left[1+\frac{\alpha}{24}(i \omega h)^{2}\right. \\
&\left.\quad+\left(\frac{\alpha^{2}}{1152}-\frac{\alpha}{2880}\right)(i \omega h)^{4}+\mathcal{O}(|i \omega h|)^{6}\right],
\end{aligned}
$$

similarly, we also have

$$
\begin{aligned}
\mathscr{F} & \left\{\left(1+\frac{\alpha}{24} \delta_{x}^{2}\right){ }_{\mathrm{RL}} D_{-\infty, x}^{\alpha} f(x), \omega\right\} \\
& =\mathscr{F}\left\{\left[1+\frac{\alpha}{24}\left(h^{2} \frac{d^{2}}{d x^{2}}+\mathcal{O}\left(h^{4}\right)\right)\right] \mathrm{RL} D_{-\infty, x}^{\alpha} f(x), \omega\right\} \\
& =(i \omega)^{\alpha} \widehat{f}(\omega)\left[1+\frac{\alpha}{24}(i \omega h)^{2}+\mathcal{O}\left(h^{4}\right)\right] .
\end{aligned}
$$

If we denote

$$
\begin{aligned}
\widehat{\phi}(\omega, h)= & \mathscr{F}\left\{\frac{C^{\Delta_{h}^{\alpha} f(x)}}{h^{\alpha}}, \omega\right\} \\
& -\mathscr{F}\left\{\left(1+\frac{\alpha}{24} \delta_{x}^{2}\right){ }_{\mathrm{RL}} D_{-\infty, x}^{\alpha} f(x), \omega\right\},
\end{aligned}
$$

then, from the above equation and Lemma 3, we have

$$
|\widehat{\phi}(\omega, h)| \leq C_{1} h^{4}\left|(i \omega)^{\alpha+4} \widehat{f}(\omega)\right| .
$$

Note that the condition $\mathscr{F}\left({ }_{\mathrm{RL}} D_{-\infty, x}^{\alpha+4} f(x), \omega\right) \in L_{1}(\mathbb{R})$; then we obtain

$$
\begin{aligned}
& \left|\frac{{ }_{C} \Delta_{h}^{\alpha} f(x)}{h^{\alpha}}-\left(1+\frac{\alpha}{24} \delta_{x}^{2}\right){ }_{\mathrm{RL}} D_{-\infty, x}^{\alpha} f(x)\right| \\
& \quad=|\phi(\omega, h)|=\frac{1}{2 \pi}\left|\int_{\mathbb{R}} \exp (i \omega h) \widehat{\phi}(\omega, h) d \omega\right| \\
& \quad \leq \frac{1}{2 \pi} \int_{\mathbb{R}}|\widehat{\phi}(\omega, h)| d \omega \\
& \leq \frac{C_{1}}{2 \pi}\left(\int_{\mathbb{R}}\left|(i \omega)^{\alpha+4} \widehat{f}(\omega)\right| d \omega\right) h^{4} \\
& \quad=\frac{C_{1}}{2 \pi}\left(\int_{\mathbb{R}}\left|\mathscr{F}\left({ }_{\mathrm{RL}} D_{-\infty, x}^{\alpha+4} f(x)\right)\right| d \omega\right) h^{4} \\
& \leq C h^{4}=\mathcal{O}\left(h^{4}\right),
\end{aligned}
$$

where $C=C_{1} C_{2} / 2 \pi$. This finishes the proof.

Remark 5. (1) When $\alpha=1$, (7) becomes the following average-central difference scheme for the first-order derivative:

$$
\frac{d f(x)}{d x}=\frac{1}{h} \frac{f(x+(1 / 2) h)-f(x-(1 / 2) h)}{1+(1 / 24) \delta_{x}^{2}}+\mathcal{O}\left(h^{4}\right) .
$$

(2) When $\alpha=2$, (7) becomes the following averagecentral difference scheme for the second-order derivative:

$$
\frac{d^{2} u(x)}{d x^{2}}=\frac{1}{h^{2}} \frac{f(x+h)-2 f(x)+f(x-h)}{1+(1 / 12) \delta_{x}^{2}}+\mathcal{O}\left(h^{4}\right) .
$$


TABLE 1: The numerical results of Example 6 by difference Scheme (7).

\begin{tabular}{|c|c|c|c|}
\hline$\alpha$ & $h$ & The absolute error & The convergence order \\
\hline \multirow{4}{*}{0.2} & $1 / 10$ & $1.507793 e-006$ & - \\
\hline & $1 / 20$ & $9.410104 e-008$ & 4.0021 \\
\hline & $1 / 40$ & $5.876619 e-009$ & 4.0012 \\
\hline & $1 / 80$ & $3.671357 e-010$ & 4.0006 \\
\hline \multirow{4}{*}{0.4} & $1 / 10$ & $2.250905 e-006$ & - \\
\hline & $1 / 20$ & $1.402929 e-007$ & 4.0040 \\
\hline & $1 / 40$ & $8.755965 e-009$ & 4.0020 \\
\hline & $1 / 80$ & $5.468621 e-010$ & 4.0010 \\
\hline \multirow{4}{*}{0.6} & $1 / 10$ & $2.154827 e-006$ & - \\
\hline & $1 / 20$ & $1.342424 e-007$ & 4.0047 \\
\hline & $1 / 40$ & $8.377252 e-009$ & 4.0022 \\
\hline & $1 / 80$ & $5.231917 e-010$ & 4.0011 \\
\hline \multirow{4}{*}{0.8} & $1 / 10$ & $1.313317 e-006$ & - \\
\hline & $1 / 20$ & $8.185957 e-008$ & 4.0039 \\
\hline & $1 / 40$ & $5.110355 e-009$ & 4.0017 \\
\hline & $1 / 80$ & $3.192508 e-010$ & 4.0007 \\
\hline \multirow{4}{*}{1.2} & $1 / 10$ & $1.371602 e-006$ & - \\
\hline & $1 / 20$ & $8.576212 e-008$ & 3.9994 \\
\hline & $1 / 40$ & $5.363498 e-009$ & 3.9991 \\
\hline & $1 / 80$ & $3.354046 e-010$ & 3.9992 \\
\hline \multirow{4}{*}{1.4} & $1 / 10$ & $2.337915 e-006$ & - \\
\hline & $1 / 20$ & $1.464272 e-007$ & 3.9970 \\
\hline & $1 / 40$ & $9.165078 e-009$ & 3.9979 \\
\hline & $1 / 80$ & $5.734311 e-010$ & 3.9985 \\
\hline \multirow{4}{*}{1.6} & $1 / 10$ & $2.507873 e-006$ & - \\
\hline & $1 / 20$ & $1.572017 e-007$ & 3.9958 \\
\hline & $1 / 40$ & $9.842569 e-009$ & 3.9974 \\
\hline & $1 / 80$ & $6.154188 e-010$ & 3.9994 \\
\hline \multirow{4}{*}{1.8} & $1 / 10$ & $1.690729 e-006$ & - \\
\hline & $1 / 20$ & $1.059074 e-007$ & 3.9968 \\
\hline & $1 / 40$ & $6.627397 e-009$ & 3.9982 \\
\hline & $1 / 80$ & $4.135963 e-010$ & 4.0021 \\
\hline
\end{tabular}

For the right Riemann-Liouville derivative, we can also obtain the following fourth-order difference scheme by using the same method:

$$
{ }_{\mathrm{RL}} D_{x,+\infty}^{\alpha} f(x)=\frac{1}{h^{\alpha}} \frac{{ }_{C} \widetilde{\Delta}_{h}^{\alpha} f(x)}{1+(\alpha / 24) \delta_{x}^{2}}+\mathcal{O}\left(h^{4}\right),
$$

where

$$
{ }_{C} \widetilde{\Delta}_{h}^{\alpha} f(x)=\sum_{j=0}^{\infty}(-1)^{j}\left(\begin{array}{c}
\alpha \\
j
\end{array}\right) f\left(x+\left(j-\frac{\alpha}{2}\right) h\right) .
$$

Combing (7) and (15), one can easily get a fourth-order compact difference scheme for Riesz derivative as follows:

$$
\begin{aligned}
\frac{\partial^{\alpha} f(x)}{\partial|x|^{\alpha}}= & -\frac{1}{2 \cos (\pi \alpha / 2) h^{\alpha}} \frac{{ }_{C} \Delta_{h}^{\alpha} f(x)+{ }_{C} \tilde{\Delta}_{h}^{\alpha} f(x)}{1+(\alpha / 24) \delta_{x}^{2}} \\
& +\mathcal{O}\left(h^{4}\right) .
\end{aligned}
$$

\begin{tabular}{|c|c|c|c|}
\hline$\alpha$ & $h$ & The absolute error & The convergence order \\
\hline \multirow{4}{*}{0.2} & $1 / 10$ & $1.009205 e-006$ & - \\
\hline & $1 / 20$ & $6.374128 e-008$ & 3.9848 \\
\hline & $1 / 40$ & $3.988170 e-009$ & 3.9984 \\
\hline & $1 / 80$ & $2.491356 e-010$ & 4.007 \\
\hline \multirow{4}{*}{0.4} & $1 / 10$ & $2.982868 e-006$ & - \\
\hline & $1 / 20$ & $1.886698 e-007$ & 3.9828 \\
\hline & $1 / 40$ & $1.180691 e-008$ & 3.9982 \\
\hline & $1 / 80$ & $7.375345 e-010$ & 4.0008 \\
\hline \multirow{4}{*}{0.6} & $1 / 10$ & $6.568541 e-006$ & - \\
\hline & $1 / 20$ & $4.168414 e-007$ & 3.9780 \\
\hline & $1 / 40$ & $2.612079 e-008$ & 3.9962 \\
\hline & $1 / 80$ & $1.632646 e-009$ & 3.9999 \\
\hline \multirow{4}{*}{0.8} & $1 / 10$ & $1.281483 e-005$ & - \\
\hline & $1 / 20$ & $8.158970 e-007$ & 3.9733 \\
\hline & $1 / 40$ & $5.120258 e-008$ & 3.9941 \\
\hline & $1 / 80$ & $3.202608 e-009$ & 3.9989 \\
\hline \multirow{4}{*}{1.2} & $1 / 10$ & $4.091054 e-005$ & - \\
\hline & $1 / 20$ & $2.613444 e-006$ & 3.9684 \\
\hline & $1 / 40$ & $1.642689 e-007$ & 3.9918 \\
\hline & $1 / 80$ & $1.028262 e-008$ & 3.9978 \\
\hline \multirow{4}{*}{1.4} & $1 / 10$ & $6.933668 e-005$ & - \\
\hline & $1 / 20$ & $4.429158 e-006$ & 3.9685 \\
\hline & $1 / 40$ & $2.783972 e-007$ & 3.9918 \\
\hline & $1 / 80$ & $1.742681 e-008$ & 3.9978 \\
\hline \multirow{4}{*}{1.6} & $1 / 10$ & $1.145559 e-004$ & - \\
\hline & $1 / 20$ & $7.311853 e-006$ & 3.9697 \\
\hline & $1 / 40$ & $4.594486 e-007$ & 3.9923 \\
\hline & $1 / 80$ & $2.875631 e-008$ & 3.9980 \\
\hline \multirow{4}{*}{1.8} & $1 / 10$ & $1.850464 e-004$ & - \\
\hline & $1 / 20$ & $1.179730 e-005$ & 3.9714 \\
\hline & $1 / 40$ & $7.409938 e-007$ & 3.9929 \\
\hline & $1 / 80$ & $4.637042 e-008$ & 3.9982 \\
\hline
\end{tabular}

TABLE 2: The numerical results of Example 7 by difference Scheme (17).

\section{Numerical Examples}

In order to verify the proposed numerical schemes for the Riemann-Liouville and Riesz derivatives, we give the following two numerical examples. And the numerical results show that the numerical schemes are efficient.

Example 6. Consider the function $f(x)=x^{4}, x \in[0,1]$. The left Riemann-Liouville derivative of the function $f(x)$ is

$$
{ }_{\mathrm{RL}} D_{0, x}^{\alpha} f(x)=\frac{24}{\Gamma(5-\alpha)} x^{4-\alpha} .
$$


The absolute error and convergence order by the numerical scheme (7) are shown in Table 1.

Example 7. Consider the function $f(x)=x^{4}(1-x)^{4}, x \in$ $[0,1]$. The Riesz derivative of the function $f(x)$ is

$$
\begin{aligned}
\frac{\partial^{\alpha} f(x)}{\partial|x|^{\alpha}}= & -\sec \left(\frac{\pi}{2} \alpha\right) \\
& \times\left\{\frac{12}{\Gamma(5-\alpha)}\left[x^{4-\alpha}+(1-x)^{4-\alpha}\right]\right. \\
& -\frac{240}{\Gamma(6-\alpha)}\left[x^{5-\alpha}+(1-x)^{5-\alpha}\right] \\
& +\frac{2160}{\Gamma(7-\alpha)}\left[x^{6-\alpha}+(1-x)^{6-\alpha}\right] \\
& -\frac{10080}{\Gamma(8-\alpha)}\left[x^{7-\alpha}+(1-x)^{7-\alpha}\right] \\
& \left.+\frac{20160}{\Gamma(9-\alpha)}\left[x^{8-\alpha}+(1-x)^{8-\alpha}\right]\right\} .
\end{aligned}
$$

The absolute error and convergence order by the numerical scheme (17) are shown in Table 2.

\section{Conclusion}

In this paper, we build two new finite difference schemes for the Riemann-Liouville and Riesz derivatives, respectively. The convergence orders of the difference schemes are proved by Fourier transform method. Finally, numerical experiments have been carried out to support the theoretical claims.

\section{Conflict of Interests}

The authors declare that there is no conflict of interests regarding the publication of this paper.

\section{Acknowledgment}

The work was partially supported by the Tianshui Normal University “QingLan” Talent Engineering Funds.

\section{References}

[1] B. L. Guo, X. K. Pu, and F. H. Huang, Fractional Partial Differential Equations and Their Numerical Solutions, Science Press, Beijin, China, 2011.

[2] K. B. Oldham and J. Spanier, The Fractional Calculus: Theory and Applications of Differentiation and Integration to Arbitrary Order, Academic Press, New York, NY, USA, 1974.

[3] I. Podlubny, Fractional Differential Equations, vol. 198 of Mathematics in Science and Engineering, Academic Press, San Diego, Calif, USA, 1999.

[4] M. H. Chen and W. H. Deng, "Fourth order accurate scheme for the spacefractional diffusion equations," SIAM Journal on Numerical Analysis. In press.

[5] M. H. Chen and W. H. Deng, "Fourth order difference approximations for space Riemann-Liouville derivatives based on weighted and shifted Lubich difference operators," Communications in Computational Physics. In press.

[6] K. Diethelm, N. J. Ford, A. D. Freed, and Yu. Luchko, "Algorithms for the fractional calculus: a selection of numerical methods," Computer Methods in Applied Mechanics and Engineering, vol. 194, no. 6-8, pp. 743-773, 2005.

[7] C. Li, A. Chen, and J. Ye, "Numerical approaches to fractional calculus and fractional ordinary differential equation," Journal of Computational Physics, vol. 230, no. 9, pp. 3352-3368, 2011.

[8] D. A. Murio, "On the stable numerical evaluation of Caputo fractional derivatives," Computers \& Mathematics with Applications, vol. 51, no. 9-10, pp. 1539-1550, 2006.

[9] T. Miyakoda, "Discretized fractional calculus with a series of Chebyshev polynomial," Electronic Notes in Theoretical Computer Science, vol. 225, pp. 239-244, 2009.

[10] Z. Odibat, "Approximations of fractional integrals and Caputo fractional derivatives," Applied Mathematics and Computation, vol. 178, no. 2, pp. 527-533, 2006.

[11] I. Podlubny, A. Chechkin, T. Skovranek, Y. Chen, and B. M. V. Jara, "Matrix approach to discrete fractional calculus. II. Partial fractional differential equations," Journal of Computational Physics, vol. 228, no. 8, pp. 3137-3153, 2009.

[12] E. Sousa, "How to approximate the fractional derivative of order $1<\alpha \leq 2$," International Journal of Bifurcation and Chaos in Applied Sciences and Engineering, vol. 22, no. 4, Article ID 1250075, 13 pages, 2012.

[13] W. Y. Tian, H. Zhou, and W. H. Deng, "A class of second order difference approximation for solving space fractional diffusion equations," http://arxiv.org/abs/1201.5949.

[14] R. F. Wu, H. F. Ding, and C. P. Li, "Determination of coefficients of high-order schemes for Riemann-Liouville derivative," The Scientific World Journal, vol. 2014, Article ID 402373, 21 pages, 2014.

[15] A. A. Kilbas, H. M. Srivastava, and J. J. Trujillo, Theory and Applications of Fractional Differential Equations, Elsevier, Amsterdam, The Netherlands, 2006.

[16] V. J. Ervin and J. P. Roop, "Variational formulation for the stationary fractional advection dispersion equation," Numerical Methods for Partial Differential Equations, vol. 22, no. 3, pp. 558576, 2006.

[17] V. K. Tuan and R. Gorenflo, "Extrapolation to the limit for numerical fractional differentiation," Zeitschrift für Angewandte Mathematik und Mechanik, vol. 75, no. 8, pp. 646-648, 1995. 


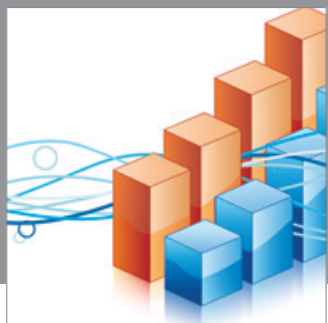

Advances in

Operations Research

mansans

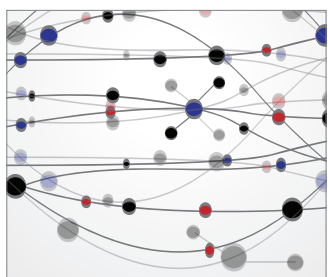

The Scientific World Journal
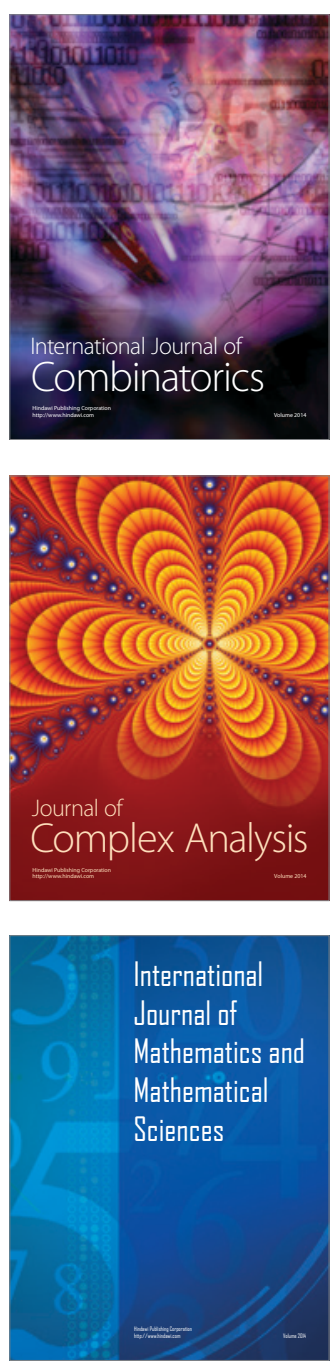
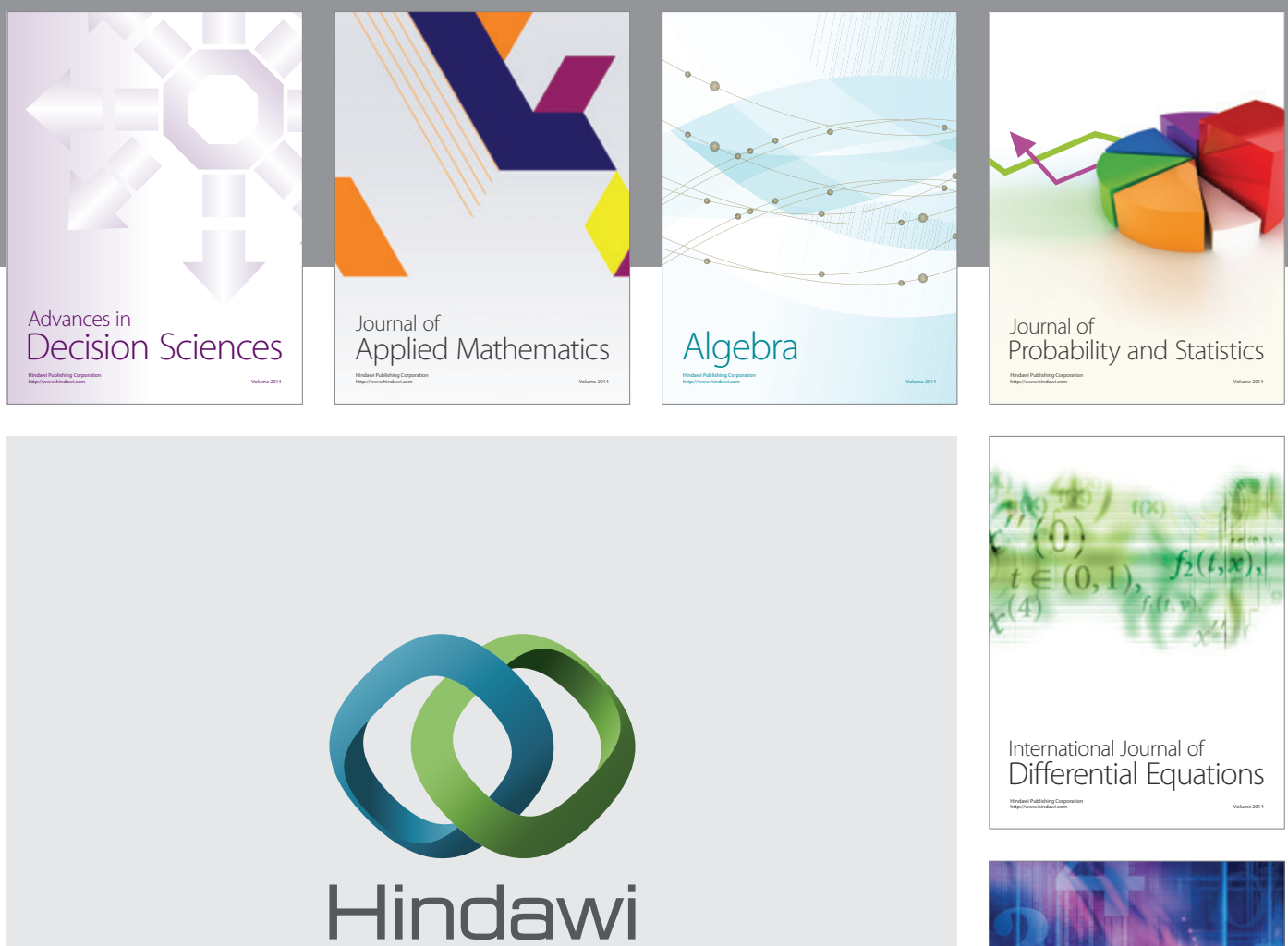

Submit your manuscripts at http://www.hindawi.com
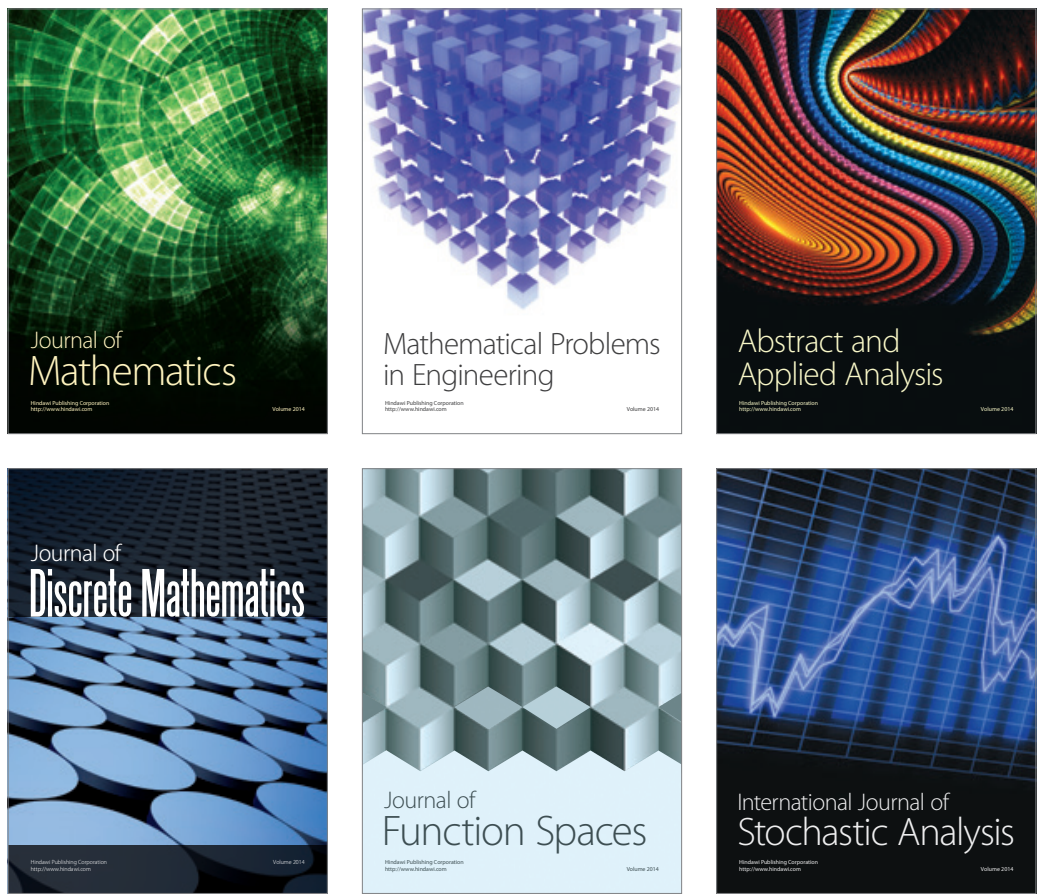

Journal of

Function Spaces

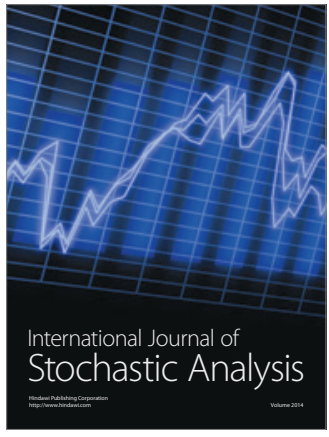

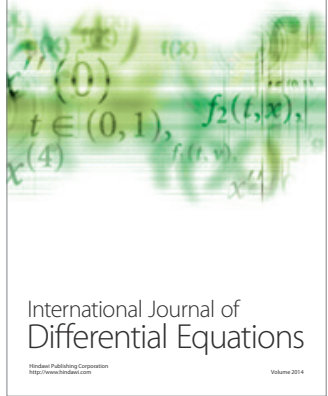
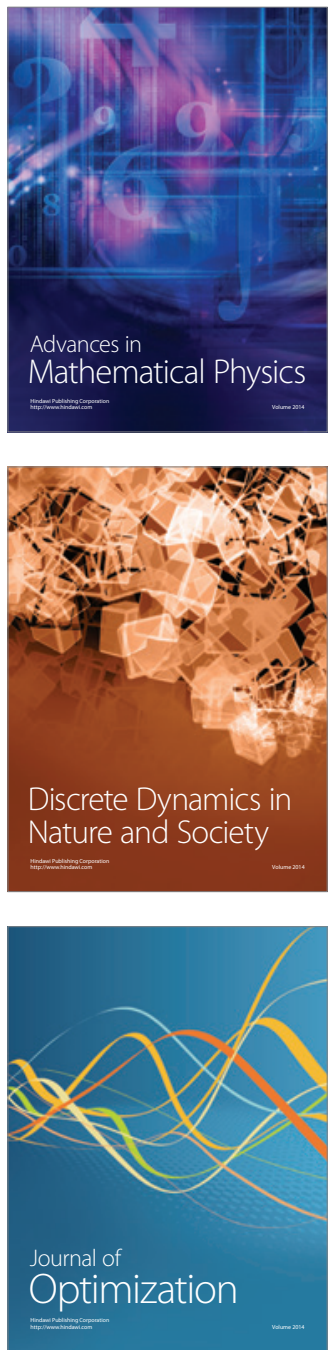